\title{
DÜBLIN
}

Technological University Dublin

ARROW@TU Dublin

\section{High Quality Millimeter Wave Generation via Stimulated Brillouin Scattering}

\author{
Marcus Junker \\ Deutsche Telekom Fachhochschule Leipzig \\ Thomas Schneider \\ Deutsche Telekom Fachhochschule Leipzig \\ Kai-Uwe Lauterbach \\ Deutsche
}

See next page for additional authors

Follow this and additional works at: https://arrow.tudublin.ie/engschececon

Part of the Electrical and Computer Engineering Commons

\section{Recommended Citation CLEO.2007.4452356 \\ This Conference Paper is brought to you for free and open access by the School of Electrical and Electronic Engineering at ARROW@TU Dublin. It has been accepted for inclusion in Conference papers by an authorized administrator of ARROW@TU Dublin. For more information, please contact arrow.admin@tudublin.ie, aisling.coyne@tudublin.ie,gerard.connolly@tudublin.ie.}

Junker, M. et al. 92007) High quality millimeter wave generation via stimulated Brillouin scattering. CLEO 2007: Conference on Lasers and Electro-Optics, pp.1-2. Baltimore, 6-11 May, 2007, doi:10.1109/

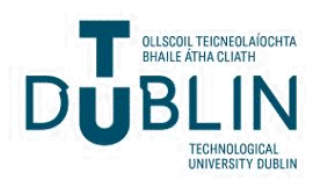




\section{Authors}

Marcus Junker, Thomas Schneider, Kai-Uwe Lauterbach, Ronny Henker, Max Ammann, and Andreas Schwarzbacher 


\title{
High Quality Millimeter Wave Generation via Stimulated Brillouin Scattering
}

\author{
Markus Junker, Thomas Schneider, Kai-Uwe Lauterbach, Ronny Henker \\ Deutsche Telekom AG, Fachhochschule Leipzig, Gustav-Freytag Straße 43-45, 02477 Leipzig, Germany \\ E-Mail: junker@fh-telekom-leipzig.de \\ Max J. Ammann, Andreas T. Schwarzbacher \\ School of Electronic \& Communications Engineering, Dublin Institute of Technology, Dublin, Ireland
}

\begin{abstract}
A new and simple method for the generation and modulation of Millimeter waves is presented. Based on frequency upconversion via Stimulated Brillouin Scattering, it is very flexible in its output frequency and modulation bandwidth. (C2007 Optical Society of America
\end{abstract}

OCIS codes: (190.4370) Nonlinear optics, fibers; (290.5900) Scattering, stimulated Brillouin

\section{Introduction}

The growing demand for wireless gigabit networks has strongly affected the research interests of new transmission systems. Recently, a $10 \mathrm{Gbit} / \mathrm{s}$ wireless transmission link at $125 \mathrm{GHz}$ verified the great potential of millimeter wave (mm-waves) photonics [1], as a prospective field of investigation.

Several optical generation techniques of mm-waves have been verified in the past. Ref. [2] and [3] reflect only a small excerpt. Most of them are based on the heterodyning of two phase correlated frequency components in a photo detector. Besides a high level of complexity and sensitivity to environmental influences, a high frequency microwave synthesizer is required limit these techniques. However, the application of Stimulated Brillouin Scattering (SBS) as a narrow band amplifier offered new opportunities in the field of mm-wave generation [3]. SBS occurs even at low power levels and has low phase noise properties [5][6]. On the other hand the gain bandwidth lies in the range of approximately $35 \mathrm{MHz}$ for Standard Single Mode Fibers (SSMF) and limits its applicability [7].

In this paper we demonstrate a new and simple mm-wave generation method that requires neither a synthesizer higher than $9.8 \mathrm{GHz}$ nor other unconventional optical components. For modulation processes it is independent of the SBS gain bandwidth. The method is based on the superposition between the modulated carrier and a sideband amplified by SBS.

\section{Experiment}

The half of the light of a fiber laser (Signal Laser: line width $<1 \mathrm{kHz}$, wavelength $\sim 1550.12 \mathrm{~nm}$, power: $24 \mathrm{~mW}$ ) is modulated by a Mach-Zehnder modulator (MZM). The MZM is driven by a $9.8 \mathrm{GHz}$ continuous wave source in the lower quadratic operation mode to realize double sideband suppressed carrier modulation. A high modulation power $(35.5 \mathrm{dBm})$ applied to the MZM generates sidebands up to the order of 6 . This frequency comb is launched into a $50 \mathrm{~km}$ Standard Single Mode Fiber (SSMF) via a $3 \mathrm{~dB}$ coupler. The other $50 \%$ of the signal laser light is amplified by an Erbium Doped Fiber Amplifier to a power level of $12 \mathrm{dBm}$ and is sent into the same SSMF as one can see in Fig. 1.

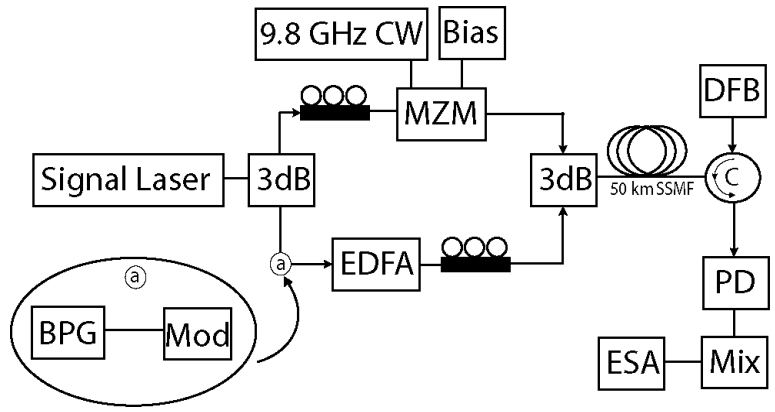

\author{
CW: Continuous Wave \\ MZM: Mach Zehnder Modulator \\ EDFA: Erbium Doped Fiber Amplifier \\ Mod:Modulator \\ BPG: Bit Pattern Generator \\ DFB:Distributed Feedback \\ PD:Photo Detector \\ Mix:External Mixer \\ ESA:Electrical Spectrum Analyzer \\ SSMF:Standard Single Mode Fiber \\ C:Optical Circulator
}

Fig. 1. Experimental set up of the Millimeter wave generator. Inset (a): A possible modulation implementation 


\section{CFD2.pdf}

The pump wave (power: $5.5 \mathrm{~mW}$ ) of a Distributed Feedback laser diode generates a backward scattered Brillouin gain that is downshifted in frequency. The pump system is driven under the Brillouin threshold. The gain frequency is adjusted in such a manner that the Brillouin gain amplifies one sideband of the frequency comb. In this paper we show a $27.1 \mathrm{~dB}$ amplification of the $5^{\text {th }}$ harmonic. The two phase correlated frequency components (optical carrier and the amplified sideband) are coupled out via an optical circulator (see Fig. 2 (left)) and are heterodyned in a $110 \mathrm{GHz}$ photo detector (PD). The output current of the PD follows the fading frequency between the two components which is $49 \mathrm{GHz}$ in our setup. Carrier modulation can be applied very simply by adding an adequate modulator into the set up as demonstrated in Fig. 1 (inset a). The idea of modulating the optical carrier and hence the modulation of the mm-wave was given by Park [8]. Since we modulate the carrier which is not affected by SBS we see no limitation concerning the modulation bandwidth, neither due to a narrow Brillouin bandwidth nor to any other restrictions.

\section{Results}

Spectrum measurements of a $49 \mathrm{GHz}$ Lorentzian shaped millimeter wave carrier can be seen in Fig. 2 (right). The optical input power of the PD is $-1.05 \mathrm{dBm}$. As one can see in Fig. 2 (right) the carrier has a signal-to-noise ratio of $70 \mathrm{~dB}$, a $3 \mathrm{~dB}$ bandwidth of approximately $500 \mathrm{~Hz}$ and a magnitude of $-34.1 \mathrm{dBm}$. Due to the natural attenuation in the fiber all other optical sidebands are decreased in their magnitude, which avoids additional electrical frequency components at the output of the PD. Of course, by using other frequency comb generation methods such as phase modulation, for instance, sidebands of higher orders and hence higher frequencies can be generated. A power fluctuation of $\pm 0.8 \mathrm{~dB}$ and a frequency fluctuation of $\pm 75 \mathrm{mHz}$ over a $6 \mathrm{~h}$ time frame was observed. Preliminary phase noise measurements let us expect a value of $-95 \mathrm{dBc} / \mathrm{Hz}$ at $10 \mathrm{kHz}$ offset from the carrier.
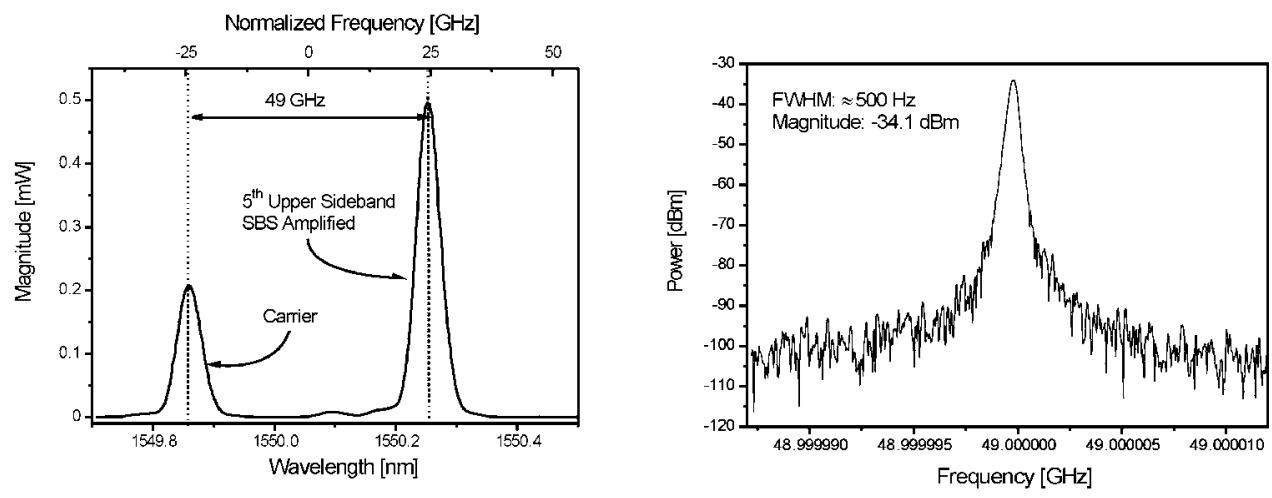

Fig. 2. Left: Optical spectrum at the circulator output; Right: Spectral analysis of the $49 \mathrm{GHz}$ carrier signal

\section{Summary}

In this paper a new and simple method for the mm-wave generation and its modulation is verified. The maximum achievable frequency depends only on the bandwidth of the photo detector. Carrier modulation can be simply implemented into the setup and, to our knowledge, there is no obvious limitation to the modulation bandwidth.

\section{References}

[1] A. Hirata, T. Kosugi, H. Takahashi, R. Yamaguchi, F. Nakajima, et. al., "120-GHz-Band Millimeter-Wave Photonic Wireless Link for 10-Gb/s Data Transmission," IEEE TMTT 54, 1937-1944 (2006).

[2] D. Wake, C. R. Lima, P. A. Davis, "Optical generation of millimetre-wave signals for fiber-radio systems using a dual mode DFB semiconductor laser," IEEE TMTT 43, 2270-2276 (1996).

[3] U. Gliese, T. N. Nielsen, M. Bruun, et. al., "A wideband heterodyne optical phase locked loop for generation of 3-18 GHz microwave carriers," IEEE Phot. Techn. Lett. 4, 936-938, (1992).

[4] X. S. Yao, "Brillouin Selective Sideband Amplification of Microwave Photonic Signals," IEEE Phot. Techn. Lett. 10, 138-140 (1998).

[5] M. Junker, M. J. Ammann, A. T. Schwarzbacher, J. Klinger, K.-U. Lauterbach, Th. Schneider, "A Comparative Test of Brillouin Amplification and Erbium Doped Fiber Amplification for the Generation of Millimeter Waves with Low Phase Noise Properties," IEEE TMTT 54, 1576-1581 (2006).

[6] Th. Schneider, M. Junker, K.-U. Lauterbach, "Theoretical and experimental investigation of Brillouin scattering for the generation of millimeter waves," JOSA B 23, 1012-1019, (2006).

[7] T. Schneider, Nonlinear Optics in Telecommunications, (Springer-Verlag, Berlin Heidelberg New York, 2004), pp. 267-296.

[8] C.S. Park, Personal Communication. 